COMMUNICATIONS IN

ANALYSIS AND GEOMETRY

Volume 12, Number 1, 213-232, 2004

\title{
Convex Hypersurfaces of Prescribed Weingarten Curvatures
}

\author{
Weimin Sheng, Neil Trudinger, and Xu-Jia Wang
}

In this paper we study the existence of closed convex hypersurfaces in the Euclidean space $\mathbb{R}^{n+1}$ with a Weingarten curvature prescribed as a function of their unit normal.

\section{Introduction.}

In this paper we study the existence of closed convex hypersurfaces in the Euclidean space $\mathbb{R}^{n+1}$ such that a Weingarten curvature, regarded as a function of their unit normal, is equal to a given positive function on the unit sphere $S^{n}$. Two classical problems of this type, namely the Christoffel problem [6] and the Minkowski problem [16], which address respectively the cases of harmonic and Gauss curvature, were completely solved in the 1970's $[7,9,17]$. Besides the harmonic and Gauss curvatures, the most interesting Weingarten curvatures are probably the mean curvature, or more generally the $k$-curvatures $(1 \leq k \leq n)$

$$
f(\kappa)=\sigma_{k}(\kappa)=\sum_{i_{1}<\cdots<i_{k}} \kappa_{i_{1}} \cdots \kappa_{i_{k}},
$$

and the norm of the second fundamental form

$$
f(\kappa)=\left(\kappa_{1}^{2}+\cdots+\kappa_{n}^{2}\right)^{1 / 2}
$$

where $\kappa=\left(\kappa_{1}, \cdots, \kappa_{n}\right)$ denotes the principal curvatures of the hypersurface $\mathcal{M}$. We will prove

Theorem 1.1. Let $f$ be the Weingarten curvatures given in (1.1) or (1.2). Then for any positive function $\varphi \in C^{2}\left(S^{n}\right)$, there exists a linear function a on $S^{n}$ such that the equation

$$
f(\kappa)=\varphi e^{a}
$$

\footnotetext{
${ }^{1}$ The first author was supported by the NSF of Zhejiang Province, China (No:102033). The second and third authors were supported by the Australian Research Council.
} 
has a smooth, uniformly convex solution $\mathcal{M} \subset \mathbb{R}^{n+1}$.

Theorem 1.1 extends a recent existence result in [13], where the existence was proved for the $k$-curvature (1.1) under the assumption that $\varphi$ is invariant under an automorphic group on $S^{n}$ without fixed point. One can choose the linear function $a=0$ under this assumption. Theorem 1.1 holds for more general Weingarten curvatures, see Remarks 2.2 and 2.3. The existence of convex hypersurfaces with prescribed curvatures is an area of extensive study. We refer reader to $[10,11,13,14]$ and the references therein.

The problem of prescribing the norm of the second fundamental form can be viewed in some sense as an extension of the isometric embedding problem (prescribing the first fundamental form), of which a typical problem is the Weyl problem [3], which asks whether the unit sphere $S^{2}$ with a metric of positive Gauss curvature can be isometrically embedded as a closed convex surface in $\mathbb{R}^{3}$.

The function $e^{a}$ in (1.3) can be interpreted as a balance condition for the Weingarten curvature $f(\kappa)$. Indeed a solution of $(1.3)$ is a soliton to the flow

$$
\dot{X}=\log (f(\kappa) / \varphi) .
$$

When $f(\kappa)=\sigma_{n}(\kappa)$ is the Gauss curvature, (1.4) is the logarithmic Gauss curvature flow studied in [5]. It was proved there that for any convex hypersurface $\mathcal{N}$, there is a unique constant $\theta>0$ and a unique vector $a \in \mathbb{R}^{n+1}$ such that $\mathcal{M}_{t}-t a$ converges to a convex hypersurface, which is a solution of (1.3) with $a(x)=\langle a, x\rangle$, where $\mathcal{M}_{t}$ is the solution of (1.4) with initial condition $\mathcal{M}_{t \mid t=0}=\theta \mathcal{N}$.

To prove Theorem 1.1 we will use as in [17] the support function of $\mathcal{M}$, given by

$$
h(x)=\sup \{\langle x, p\rangle: p \in \mathcal{M}\} \quad x \in S^{n},
$$

and reduce the problem to an elliptic equation for $h$ on $S^{n}$. We extend $h$ to $\mathbb{R}^{n+1}$ such that it is a homogeneous function of degree 1 . Then $h$ is convex and $\mathcal{M}$ can be recovered from $h$ by

$$
\mathcal{M}=\left\{D h(x): x \in S^{n}\right\} .
$$

Direct computation $[17,18]$ shows that the eigenvalues of the matrix $\left\{\nabla^{2} h+\right.$ $h I\}$ are the principal radii of $\mathcal{M}$, where $\nabla$ is the covariant derivative on $S^{n}$ (under a local orthonormal frame), and $I$ is the unit matrix. Denote by $\lambda=\left(\lambda_{1}, \cdots, \lambda_{n}\right)$ the eigenvalues of $\left\{\nabla^{2} h+h I\right\}$. Then equation (1.3) is equivalent to

$$
\hat{f}\left(\lambda\left(\nabla^{2} h+h I\right)(x)\right)=\psi(x) \quad \text { on } \quad S^{n}
$$


with $\psi=\left(\varphi e^{a}\right)^{-1}$, where

$$
\hat{f}(\lambda)=\left[f\left(\frac{1}{\lambda_{1}}, \cdots, \frac{1}{\lambda_{n}}\right)\right]^{-1} .
$$

Another special class of Weingarten curvatures occurs when $f(\kappa)=$ $\sigma_{k}(\lambda)$, the $k^{t h}$ elementary symmetric polynomial of $\lambda$, where $1 \leq k \leq n$, $\lambda=\left(\lambda_{1}, \cdots, \lambda_{n}\right)$, and $\lambda_{i}=\kappa_{i}^{-1}(i=1, \cdots, n)$ are the principal radii of $\mathcal{M}$. The intermediate Christoffel-Minkowski problem is to find closed convex hypersurfaces satisfying

$$
\sigma_{k}(\lambda)=\varphi,
$$

where $\varphi$ is a given positive function on the unit sphere $S^{n}$. When $k=1$ and $k=n,(1.9)$ is respectively the Christoffel and the Minkowski problem, and has been resolved in $[7,9,17]$. For the intermediate Christoffel-Minkowski problem, we have the following result.

Theorem 1.2, Let $\varphi$ be a smooth, positive function on the unit sphere $S^{n}$. Suppose $\varphi^{-1 / k}$ is convex and $\varphi$ satisfies

$$
\int_{S^{n}} x_{i} \varphi(x) d x=0, \quad \forall \quad i=1, \cdots, n+1 .
$$

Then there is a smooth, uniformly convex hypersurface $\mathcal{M}$ satisfying (1.9).

Theorem 1.2 was proved in [14] under the following additional condition.

Condition (A). There is a family of smooth, positive functions $\left\{\varphi_{t}: t \in\right.$ $[0,1]\}$, which depends continuously on $t$, such that $\varphi_{0} \equiv 1, \varphi_{1}=\varphi$, and for all $t \in[0,1], \varphi_{t}^{-1 / k}$ is convex and $\varphi_{t}$ satisfies (1.10).

Condition (A) was removed by a curvature flow method [2], which uses the a priori estimates and the analysis of asymptotic behavior of solutions. In this paper we verify this condition directly.

The operator in (1.9) is of divergence form and its linearized operator is self-adjoint. By the Aleksandrov-Fenchel inequality [18], its kernel is the linear space spanned by the linear functions $\left\{x_{1}, \cdots, x_{n+1}\right\}$, which is independent of the support function $h$. Hence (1.10) is a necessary condition for the solvability of the Christoffel-Minkowski problem. From the AleksandrovFenchel inequality one also obtains the uniqueness of solutions to (1.9).

It follows that when $k=n$, the linear function $a$ in Theorem 1.1 is uniquely determined by

$$
\int_{S^{n}} \frac{x_{i}}{\varphi(x) e^{a(x)}} d x=0 \quad i=1, \cdots, n+1 .
$$


For other Weingarten curvatures in (1.1) and (1.2), the operator $\hat{f}$ is not in divergence form and the linear functions $\left\{x_{1}, \cdots, x_{n+1}\right\}$ are in general not in the kernel of the adjoint linearized operator. Hence (1.11) is not a necessary condition for equation (1.3) anymore. See also discussions in [13]. We remark that the uniqueness of solutions for general Weingarten curvatures is open in general, except in dimension two [1].

This paper is organized as follows. In Section 2 we use a priori estimates and a degree theory, which was used in [13], to prove Theorem 1.1. The main new estimate of the paper is that for $\sup _{S^{n}}|a|$, which will be given in Section 3 . In section 4 , we verify condition (A).

\section{Proof of Theorem 1.1.}

In this section we use the degree theory to prove Theorem 1.1. We will consider the equation

$$
f(\kappa)=\varphi
$$

for more general Weingarten curvatures. We assume that $f$ is defined on the positive cone $\Gamma^{+}=\left\{\kappa \in \mathbb{R}^{n}: \kappa_{i}>0\right.$ for $\left.i=1, \cdots, n\right\}$, and satisfies the following conditions.

[F1] $f(\kappa+\mu)>f(\kappa)$, for all $\kappa \in \Gamma$ and $\mu \in \Gamma^{+}$.

[F2] $f$ is invariant under any permutation of $\left(\kappa_{1}, \cdots, \kappa_{n}\right)$.

[F3] $f(0)=0$ and for any $\kappa \in \Gamma^{+}$,

$$
f\left(\kappa_{1}, \cdots, \kappa_{n}+t\right) \rightarrow+\infty \quad \text { as } t \rightarrow+\infty .
$$

[F4] The function $\hat{f}(\lambda)$ given in (1.8) is concave on the positive cone $\Gamma^{+}$.

The norm of the second fundamental form satisfies [F1]-[F4] [10]. For the $k$-curvature (1.1), we redefine

$$
f(\kappa)=\left[\sigma_{k}(\kappa)\right]^{1 / k}
$$

so that it also satisfies [F1]-[F4]. Other Weingarten curvatures satisfying the above assumptions are, for example,

$$
f(\kappa)=\left[\sum \kappa_{1}^{\alpha_{1}} \cdots \kappa_{n}^{\alpha_{n}}\right]^{1 / k}
$$

where the sum is taken over all nonnegative integers $\alpha_{1}, \cdots, \alpha_{n}$ with $\sum \alpha_{i}=k$, see [10]. Note that the curvature (2.3) is also a function of the $k$-curvatures, given by $(2.2)$. 
For any $t \in[0,1]$, we denote

$$
F_{t}\left[\nabla^{2} h+h I\right]=(1-t) \sigma_{n}^{1 / n}(\lambda)+t \hat{f}(\lambda),
$$

where $\lambda=\lambda\left(\nabla^{2} h+h I\right)$ are the eigenvalues of the matrix $\left\{\nabla^{2} h+h I\right\}$.

Denote by $\Phi$ the set of positive, uniformly convex, $C^{3}$ smooth functions on $S^{n}$. For any positive constant $R>1$, let $\Phi_{R} \subset \Phi$ be given by

$$
\Phi_{R}=\left\{u \in C^{3}\left(S^{n}\right): u>R^{-1},\left(\nabla^{2} u+u I\right)>R^{-1} I,\|u\|_{C^{3}\left(S^{n}\right)}<R\right\} .
$$

We also denote by $\Phi^{0}$ ( $\Phi_{R}^{0}$, resp.) the set of functions $u \in \Phi\left(u \in \Phi_{R}\right.$, resp.) which satisfy

$$
\int_{S^{n}} x_{i} u(x)=0 \quad \forall i=1, \cdots, n+1 .
$$

Note that if $u \in C^{2}\left(S^{n}\right)$ satisfies $\left(\nabla^{2} u+u I\right)>0$, then $u$ is convex on $S^{n}$ and it is a support function of a convex hypersurface $\mathcal{M}$ given by (1.6).

Remark 2.1. For any $u \in C^{2}\left(S^{n}\right)$, there is a unique linear function $a$ defined in $\mathbb{R}^{n+1}$ with $a(0)=0$, such that $u+a$ satisfies $(2.5)$. Obviously the function $a$ depends continuously on $u$. If $u$ is the support function of a convex hypersurface $\mathcal{M}$ and it satisfies (2.5), one easily verifies that the origin is located in the interior of $\mathcal{M}$.

Lemma 2.1. For any positive functions $\psi, v \in C^{2}\left(S^{n}\right)$, and for each $t \in$ $[0,1]$, there is a unique solution $h \in C^{3, \alpha}\left(S^{n}\right)(\alpha \in(0,1))$ to the equation

$$
F\left[\nabla^{2} h+v I\right]=e^{h-v} \psi
$$

such that the matrix $\left\{\nabla^{2} h+v I\right\}>0$, where $F\left[\nabla^{2} h+v I\right]=F_{t}\left[\nabla^{2} h+v I\right]$ and

$$
F\left[\nabla^{2} h+v I\right]=(1-t) \sigma_{n}^{1 / n}\left(\lambda\left(\nabla^{2} h+v I\right)\right)+t \hat{f}\left(\lambda\left(\nabla^{2} h+v I\right)\right) .
$$

Proof. The proof is similar to that in [13], where the case $F\left[\nabla^{2} h+v I\right]=$ $\sigma_{k}^{1 / k}\left(\lambda\left(\nabla^{2} h+v I\right)\right)$ is considered (with $e^{h-v}$ replaced by $\left.h / v\right)$. We sketch the proof and indicate some minor changes.

First we prove that there is a positive constant $C>0$ such that

$$
-C \leq h \leq C .
$$

Indeed, suppose $h$ attains a maximum at $x_{0}$. Then at $x_{0}$,

$$
e^{h-v} \psi=F\left[\nabla^{2} h+v I\right] \leq F[v I],
$$


which yields the second inequality in (2.7). Similarly we obtain the first inequality.

Next we prove

$$
\sup \left\{\lambda_{i}\left(\nabla^{2} h+v I\right)(x): x \in S^{n}, i=1, \cdots, n\right\} \leq C,
$$

Suppose the supremum in (2.8) is attained at $x_{0}$ and $i=1$. By choosing a proper local orthonormal frame we may suppose that the matrix $\left\{h_{i j}\right\}=$ $\left\{\nabla_{i} \nabla_{j} h\right\}$ is diagonal at $x_{0}$, where $\nabla_{i}=\nabla_{e_{i}}$ denotes the covariant derivative. Denote $F^{i j}=\frac{\partial}{\partial w_{i j}} F\left[w_{i j}\right]$. By $[\mathrm{F} 1],\left\{F^{i j}\right\}$ is positive definite. Hence by exchanging derivatives we have

$$
\begin{aligned}
0 & \geq F^{i i} \nabla_{i}^{2}\left(h_{11}+v\right) \\
& =F^{i i} \nabla_{1}^{2}\left(h_{i i}+v\right)-2 F^{i i}\left(h_{i i}+v\right)+\left(2 h_{11}+2 v-v_{11}+v_{i i}\right) F^{i i} .
\end{aligned}
$$

By the concavity of $F$ we have

$$
\begin{aligned}
F^{i i} \nabla_{1}^{2}\left(h_{i i}+v\right) & \geq \nabla_{1}^{2}\left[e^{h-v} \psi\right], \\
F^{i i}\left(h_{i i}+v\right) & \leq F\left[\nabla^{2} h+v I\right]=e^{h-v} \psi .
\end{aligned}
$$

We claim that for any constant $b>0$, there exists a constant $\delta>0$, such that

$$
\sum \hat{f}_{i}(\lambda) \geq \delta \quad \forall \lambda \in \Gamma_{b}
$$

where $\hat{f}_{i}(\lambda)=\frac{\partial}{\partial \lambda_{i}} \hat{f}(\lambda)$ and $\Gamma_{b}=\{\lambda \in \Gamma: \hat{f}(\lambda)=b\}$. Indeed, denote $d_{b}=\sup \left\{\left|\lambda-\lambda^{\prime}\right|: \lambda \in \Gamma_{b}, \lambda^{\prime} \in \partial \Gamma^{+}\right\}$. Then it suffices to prove $d_{2 b} \leq C$. But this follows from the facts that $\Gamma^{+}$is a convex cone and that $\hat{f}=0$ on $\partial \Gamma^{+}$by [F3].

By (2.10) we have $\sum F^{i i} \geq \delta$. Therefore if $h_{11}+2 v-v_{11}+v_{i i}>0$, we have

$$
0 \geq \nabla_{1}^{2}\left[e^{h-v} \psi\right]-e^{h-v} \psi+\delta h_{11}
$$

and so (2.8) holds. If $h_{11}+2 v-v_{11}+v_{i i} \leq 0$, we also obtain (2.8).

By assumption [F3], we have $\hat{f}(\lambda)=0$ on $\partial \Gamma^{+}$. By $(2.7), F\left[\nabla^{2} h+v I\right]$ has a positive lower bound. By (2.8), $\lambda_{i}$ are upper bounded. Hence by the continuity of the function $(1-t) \sigma_{n}^{1 / n}+t \hat{f}$, there is a positive constant $C>0$ such that

$$
\inf \left\{\lambda_{i}\left(\nabla^{2} h+v I\right)(x): x \in S^{n}, i=1, \cdots, n\right\} \geq C .
$$

Namely the principal curvatures of the corresponding convex hypersurfaces are bounded. 
With estimates (2.8) and (2.11), equation (2.6) becomes a uniformly elliptic equation. Hence by the Evans-Krylov regularity theory [12], we obtain

$$
\|h\|_{C^{3}\left(S^{n}\right)} \leq C .
$$

The existence of solutions to (2.6) then follows from the continuity method. Indeed, write (2.6) in the form

$$
\log F\left[\nabla^{2} h+v I\right]=h-v+\log \psi
$$

The linearized operator is

$$
L[u]=\sum a_{i j} u_{i j}-u .
$$

The coefficient before $u$ is negative. Hence the comparison principle holds and the operator $L$ is invertible. Hence the continuity method applies and also the solution is unique.

By Lemma 2.1 we introduce a mapping $T_{t}(t \in[0,1])$ as follows. For any positive function $v \in C^{2}\left(S^{n}\right)$, by Lemma 2.1 there is a unique solution $h$ to

$$
F_{t}\left[\nabla^{2} h+v I\right]=e^{h-v} \psi_{t}
$$

where $\psi_{t}=(1-t)+t \psi$. By Remark 2.1, there is a unique linear function $a$ with $a(0)=0$ such that $h-a$ satisfies (2.5). We define $T_{t}(v)=h-a$.

This mapping is similar to that in [13], where $u / v$ is used instead of $e^{u-v}$. Obviously $T_{t}$ is continuous in $t$. If $h$ is a fixed point of $T_{t}$, it satisfies the equation

$$
F_{t}\left[\nabla^{2} h+h I\right]=\psi_{t} e^{a} \quad \text { on } S^{n} .
$$

To prove that the fixed points of $T_{t}(t \in[0,1])$ are uniformly bounded, we need to restrict to curvature functions $(2.2)$ or (1.2).

Lemma 2.2. Suppose the curvature function $f$ is given by (2.2) or (1.2). Then there exists $R>0$ depending only on $n$, $\inf _{S^{n}} \psi$ and $\sup _{S^{n}}\left|\nabla^{2} \psi\right|$, such that if $h$ is a solution of (2.15) and $h$ satisfies (2.5), then $h \in \Phi_{R}^{0}$.

Proof. In Section 3 we will prove $\sup _{S^{n}}|a| \leq C$ for some constant $C>0$ depending only on $n, \inf _{S^{n}} \psi$ and $\sup _{S^{n}}\left|\nabla^{2} \psi\right|$. Once this is established, we can derive estimates (2.8) and (2.11), with $v$ replaced by $h$, in a similar way as above. Indeed, when $v$ is replaced by $h,(2.9)$ becomes

$$
0 \geq F^{i i} \nabla_{1}^{2}\left(h_{i i}+h\right)-F^{i i}\left(h_{i i}+h\right)+\left(h_{11}+h\right) F^{i i}
$$


and so one obtains (2.8). Note that by (2.5) and (2.8) we have $h \geq 1 / R$ for some $R>0$ depending on the constant $C$ in (2.8). We omit the details here.

By Lemmas 2.1 and 2.2, the mapping $T_{t}$ has no fixed points on the boundary of $\Phi_{R}^{0}$ when $R$ is sufficiently large. Hence the degree $\operatorname{deg}(I-$ $\left.T_{t}, \Phi_{R}^{0}, 0\right)$ is well defined if $R$ is large enough, and is independent of $t \in[0,1]$, where $I$ is the identity mapping.

When $t=0$, a fixed point of $T_{0}$ satisfies the equation

$$
\left[\sigma_{n}\left(\lambda\left(\nabla^{2} h+h I\right)\right)\right]^{1 / n}=e^{a} \quad \text { on } S^{n} .
$$

By the necessary condition (1.11), we see that if (2.16) has a solution, then the linear function $a$ is a constant. Recall that $a(0)=0$. We have $a \equiv 0$. By (2.5) and the uniqueness of solutions to the Minkowski problem, we have $h \equiv 1$. Hence $T_{0}$ has a unique fixed point.

Similarly as in [13], see also [15], one can prove that the degree $\operatorname{deg}(I-$ $\left.T_{0}, \Phi_{R}^{0}, 0\right)=-1$. Hence $\operatorname{deg}\left(I-T_{1}, \Phi_{R}^{0}, 0\right)=-1$. Namely Theorem 1.1 holds.

Remark 2.2. Although Theorem 1.1 treats the $k$-curvature and the norm of second fundamental form only, it is also true for some other Weingarten curvatures, such as (2.3). Indeed Theorem 1.1 holds for any curvature function satisfying [F1]-[F4], provided one can prove an upper bound for $\sup _{S^{n}}|a|$ for any solution $h$ of $(2.15)$.

Remark 2.3. If $\varphi$ is an even function, we can restrict ourself to the set of even functions. Then automatically $a=0$ in (2.15) and so Lemma 2.2 holds. Hence Theorem 1.1 holds for all Weingarten curvatures satisfying [F1]-[F4] if $\varphi$ is even.

\section{Proof of Lemma 2.2 .}

We have two different proofs for Lemma 2.2. One is based on the maximum principle and the other reduces to estimation on the Gauss curvature equation. We will give a detailed proof for the first case and sketch the second.

For any given $t \in[0,1]$, we denote

$$
\hat{g}(\lambda)=(1-t) \sigma_{n}^{1 / n}(\lambda)+t \hat{f}(\lambda)
$$

and $F\left[\nabla^{2} h+h I\right]=\hat{g}(\lambda)$, where $\lambda=\lambda\left(\nabla^{2} h+h I\right)=\left(\lambda_{1}, \cdots, \lambda_{n}\right)$ are the eigenvalues of $\left\{\nabla^{2} h+h I\right\}$. We will always assume that $\lambda_{1} \geq \cdots \geq \lambda_{n}$. To prove Lemma 2.2 we need only to prove 
Lemma 3.1. Suppose the curvature function $f$ is given by (2.2) or (1.2). Suppose $\psi \in C^{2}\left(S^{n}\right)$ is a positive function. If the equation

$$
F\left[\nabla^{2} h+h I\right]=e^{a} \psi(x) \quad \text { on } \quad S^{n}
$$

admits a convex solution, then

$$
\sup _{x \in S^{n}}|a(x)| \leq C
$$

for some $C>0$ depending only on $n, \inf _{S^{n}} \psi$ and $\sup _{S^{n}}\left|\nabla^{2} \psi\right|$.

To prove Lemma 3.1, we choose a proper coordinate system such that

$$
a(x)=\alpha x_{n+1}, \quad \alpha \geq 0 .
$$

Then it suffices to prove $\alpha<C$.

Let $\mathcal{M}$ be the corresponding hypersurface. Let $\Omega$ be the projection of $\mathcal{M}$ on $\left\{x_{n+1}=0\right\}$. We divide

$$
\mathcal{M}=\mathcal{M}^{+} \cup \mathcal{M}^{-} \cup \tilde{\mathcal{M}}
$$

such that the Gauss mapping image of $\mathcal{M}^{+}$and $\mathcal{M}^{-}$are respectively the upper and lower hemispheres, and $\tilde{\mathcal{M}}=\partial \mathcal{M}^{+}\left(=\partial \mathcal{M}^{-}\right)$. Then $\mathcal{M}^{+}$satisfies

$$
g(\kappa)=\varphi e^{-\alpha\left|\gamma_{n+1}\right|},
$$

where $\varphi=\psi^{-1}, \gamma_{n+1}$ is the component of the unit outer normal of $\mathcal{M}$ in $x_{n+1}$ direction,

$$
g(\kappa)=\left[\hat{g}\left(\frac{1}{\kappa_{1}}, \cdots, \frac{1}{\kappa_{n}}\right)\right]^{-1} .
$$

Similarly, $\mathcal{M}^{-}$satisfies

$$
g(\kappa)=\varphi e^{\alpha\left|\gamma_{n+1}\right|}
$$

Lemma 3.2. Let $\varepsilon_{0}=\frac{1}{2 n}$. For any large constant $\lambda_{0}>0$, there exists a constant $\alpha_{0}>0$ such that if $\alpha>\alpha_{0}$, the least eigenvalue $\lambda_{n}$ of the matrix $\left\{\nabla^{2} h+h I\right\}$ satisfies

$$
\lambda_{n} \geq \lambda_{0} \quad \forall x \in S_{\varepsilon_{0}}^{n},
$$

where $S_{\varepsilon_{0}}^{n}=S^{n} \cap\left\{x_{n+1} \geq 1-\varepsilon_{0}\right\}$.

Proof. We denote by $L=F^{i j} \nabla_{i j}^{2}$ the linearized operator of $F$, and denote $w_{i j}=h_{i j}+h \delta_{i j}, W=\sum w_{i i}$. At any fixed point, by choosing a proper 
coordinate system, we may assume $w_{i j}$ is diagonal. Then we have by the Ricci identity,

$$
W_{i i}=\left(\Sigma_{j} w_{j j}\right)_{i i}=\Delta\left(w_{i i}\right)-n w_{i i}+W
$$

Hence

$$
L(W)=F^{i i} W_{i i}=F^{i i} \Delta\left(w_{i i}\right)-n w_{i i} F^{i i}+W F^{i i} .
$$

Taking two covariant derivatives of equation (3.1) and using the concavity of $F$, we have

$$
F^{i i} \Delta\left(w_{i i}\right) \geq \Sigma_{j} \nabla_{j}^{2}\left(e^{a} \psi\right)
$$

Also by the concavity of $F$,

$$
F^{i i} w_{i i} \leq F\left[\nabla^{2} h+h I\right]=e^{a} \psi(x)
$$

We thus obtain

$$
\begin{aligned}
L(W) & \geq \sum_{i} \nabla_{i}^{2}\left(e^{a} \psi\right)-n e^{a} \psi+W \sum F^{i i} \\
& \geq-C \alpha e^{a}+W \sum F^{i i},
\end{aligned}
$$

where we assume $\alpha>1$. We claim that there exists $\theta>0$ such that

$$
\sum F^{i i} \geq \theta \frac{\hat{g}(\lambda)}{\lambda_{n}}
$$

Indeed, denote $\hat{h}(\lambda)=\left[\lambda_{1} \cdots \lambda_{n}\right]^{1 / n}$ and $h(\kappa)=[\hat{h}(\kappa)]^{-1}$, where $\kappa_{i}=1 / \lambda_{i}$, $i=1, \cdots, n$. By definition,

$$
\hat{g}(\lambda)=t \hat{f}(\lambda)+(1-t) \hat{h}(\lambda)=\frac{t}{f(\kappa)}+\frac{1-t}{h(\kappa)} .
$$

We have

$$
\hat{g}_{\lambda_{i}}(\lambda)=t f^{-2} f_{\kappa_{i}}(\kappa) \kappa_{i}^{2}+(1-t) h^{-2} h_{\kappa_{i}}(\kappa) \kappa_{i}^{2} .
$$

Note that $F^{i i}=\hat{g}_{\lambda_{i}}$. Hence (3.9) holds if

$$
\begin{aligned}
& \sum f_{i}(\kappa) \kappa_{i}^{2} \geq \theta \kappa_{n} f(\kappa), \\
& \sum h_{i}(\kappa) \kappa_{i}^{2} \geq \theta \kappa_{n} h(\kappa) .
\end{aligned}
$$

The first inequality holds as the curvature is given by (2.2) or (1.2). The second one holds with $\theta=1 / n$ as $h(\kappa)=\left[\kappa_{1} \cdots \kappa_{n}\right]^{1 / n}$. Hence (3.9) holds.

From (3.9) it follows that

$$
L(W) \geq-C \alpha e^{a}+C_{1} \frac{\lambda_{1}}{\lambda_{n}} e^{a} .
$$


Now let us suppose that $W$ attains its maximum at some point $x_{0}$. Then $L(W) \leq 0$ at $x_{0}$. Hence we have $\frac{\lambda_{1}}{\lambda_{n}} \leq C \alpha$ at $x_{0}$, i.e.,

$$
\lambda_{n} \geq \frac{\lambda_{1}}{C \alpha} .
$$

Noting that $\hat{g}$ is homogeneous of degree 1, we have, at any point on $S^{n}$,

$$
\lambda_{n}=\hat{g}\left(\lambda_{n}, \cdots, \lambda_{n}\right) / \hat{g}(1, \cdots, 1) \leq C \hat{g}\left(\lambda_{1}, \cdots, \lambda_{n}\right)=C \psi e^{\alpha x_{n+1}} .
$$

Hence at $x_{0}$ we have $\lambda_{1} \leq C \alpha e^{\alpha}$. We obtain $\sup \sum w_{i i} \leq C \alpha e^{\alpha}$ on $S^{n}$. It follows that

$$
\lambda_{1} \leq C \alpha e^{\alpha} \quad \text { on } S^{n} .
$$

Noting also that at any point in $S_{\varepsilon_{0}}^{n}$,

$$
C e^{\left(1-\varepsilon_{0}\right) \alpha} \leq \hat{g}(\lambda) \leq \hat{g}\left(\lambda_{1}, \cdots, \lambda_{1}\right)=\lambda_{1} \hat{g}(1, \cdots, 1),
$$

we have

$$
\lambda_{1} \geq C e^{\left(1-\varepsilon_{0}\right) \alpha} .
$$

On $S_{\varepsilon_{0}}^{n}$, we have the eigenvalues $\lambda_{1} \geq \cdots \geq \lambda_{n}$ and the corresponding principal curvatures $\kappa_{1}=\lambda_{1}^{-1} \leq \cdots \leq \kappa_{n}=\lambda_{n}^{-1}$. In order to prove $\lambda_{n}$ is large, we only need to prove $\kappa_{n}$ is small on $\mathcal{M}_{\varepsilon_{0}}^{+}=\left\{p \in \mathcal{M}^{+}: G(p) \in S_{\varepsilon_{0}}^{n}\right\}$, where $G$ is the Gauss mapping.

From (3.12), for any point $p_{0} \in \mathcal{M}_{\varepsilon_{0}}^{+}$, we have $\lambda_{1} \leq C \alpha e^{\alpha}$, hence

$$
\kappa_{1} \geq \varepsilon=: \frac{C}{\alpha} e^{-\alpha}
$$

If $\kappa_{n}$ is not sufficient small at $p_{0}$, we may assume for simplicity that $k_{n}=1$ at $p_{0}$. Hence by (3.4) and the estimate (3.14),

$$
g(\varepsilon, \cdots, \varepsilon, 1) \leq g\left(\kappa_{1}, \cdots, \kappa_{n-1}, \kappa_{n}\right)=\varphi e^{-\alpha\left|\gamma_{n+1}\right|} \leq C e^{-\alpha\left(1-\varepsilon_{0}\right)} .
$$

We have

$$
g\left(\kappa_{1}, \cdots, \kappa_{n}\right)=\left[\frac{1-t}{\sigma_{n}^{1 / n}(\kappa)}+\frac{t}{f(\kappa)}\right]^{-1}=\frac{f(\kappa) \sigma_{n}^{1 / n}(\kappa)}{(1-t) f(\kappa)+t \sigma_{n}^{1 / n}(\kappa)}
$$

Hence by (3.15),

$$
f(\kappa) \sigma_{n}^{1 / n}(\kappa) \leq C e^{-\alpha\left(1-\varepsilon_{0}\right)}\left[(1-t) f(\kappa)+t \sigma_{n}^{1 / n}(\kappa)\right]
$$

at $\kappa=(\varepsilon, \cdots, \varepsilon, 1)$. 
If the curvature function $f$ is given by $(2.2)$, we obtain

$$
\varepsilon^{\frac{n-1}{n}} \varepsilon^{\frac{k-1}{k}} \leq C \varepsilon \alpha e^{\alpha \varepsilon_{0}}\left[(1-t) \varepsilon^{\frac{k-1}{k}}+t \varepsilon^{\frac{n-1}{n}}\right],
$$

or equivalently

$$
C \leq \alpha e^{\alpha \varepsilon_{0}}\left[(1-t) \varepsilon^{1 / n}+t \varepsilon^{1 / k}\right] .
$$

Since $\varepsilon_{0}=\frac{1}{2 n}$ and $\varepsilon=\frac{C}{\alpha} e^{-\alpha}$, we obtain

$$
C \leq \alpha e^{-\alpha / 2 n}
$$

This is a contradiction when $\alpha$ is sufficiently large.

If the curvature function $f$ is given by (1.2), then we have

$$
\varepsilon^{\frac{n-1}{n}} \leq C \varepsilon \alpha e^{\alpha \varepsilon_{0}}\left[(1-t)+t \varepsilon^{\frac{n-1}{n}}\right]
$$

or equivalently

$$
C \leq \alpha e^{\alpha \varepsilon_{0}}\left[(1-t) \varepsilon^{1 / n}+t \varepsilon\right] .
$$

We also reach a contradiction if $\alpha$ is sufficiently large.

For the curvatures given in (2.2) and (1.2), one has $\sum F^{i i} \geq C>0$ and the estimate (3.12) follows from (3.8). Our proof of (3.12) applies to other curvature functions.

Proof of Lemma 3.1. By Lemma 3.2, if $\alpha$ is sufficiently large, so is the the least eigenvalue of the matrix $\left\{\nabla^{2} h+h I\right\}$ on the set $S_{\varepsilon_{0}}^{n}$, where $\varepsilon_{0}=\frac{1}{2 n}$. Namely all of the principal radii of $\mathcal{M}^{+}$are large, and so the principal curvatures of $\mathcal{M}^{+}$are small. It follows that the inscribed ball $B_{R}\left(x_{0}\right)$ of $\Omega$, where $\Omega$ is the projection of $\mathcal{M}^{+}$on $\left\{x_{n+1}=0\right\}$, is large.

On the other hand, consider the part $\mathcal{M}^{-}$, which is a graph of a convex function $u_{0}$ defined on $\Omega$, and satisfies equation (3.5). Let us consider the equation

$$
\begin{cases}g(\kappa)=\delta & \text { in } B_{R / 2}\left(x_{0}\right), \\ u=\max \left\{u_{0}(x): x \in B_{R / 2}\left(x_{0}\right)\right\} & \text { on } \partial B_{R / 2}\left(x_{0}\right),\end{cases}
$$

where $\delta>0$ is a constant, and $g$ is as in (3.4). Obviously (3.16) has unique solution $u_{\delta}$ which is rotationally symmetric (with center at $x_{0}$ ). Hence the graph of $u_{\delta}$ is a piece of sphere. Let $\delta_{0}$ be the largest constant such that (3.16) admits a solution. Then the graph of $u_{\delta_{0}}$ is a hemisphere. Obviously $\delta_{0} \rightarrow 0$ as $R \rightarrow \infty$. 
By equation (3.5), the function $u_{0}$ satisfies $g(\kappa) \geq \inf \psi>0$. Let $R$ be sufficiently large such that $\delta_{0}<\inf \psi$. By the boundary condition in (3.16), we have $u_{\delta_{0}} \geq u_{0}$ on $\partial B_{R / 2}\left(x_{0}\right)$ and there is a point $y_{0} \in \partial B_{R / 2}\left(x_{0}\right)$ such that $u_{\delta_{0}}\left(y_{0}\right)=u_{0}\left(y_{0}\right)$. By the comparison principle, we have $u_{\delta_{0}} \geq u_{0}$. It follows that $\partial_{\gamma} u_{0}\left(y_{0}\right) \geq \partial_{\gamma} u_{\delta_{0}}\left(y_{0}\right)=\infty$, where $\gamma$ is the unit outer normal. We reach a contradiction.

We sketch our second proof of Lemma 3.1. Let us first consider the $k$-curvature (1.1). Let $\mathcal{M}^{+}, \mathcal{M}^{-}$, and $\Omega$ be as before. Let

$$
E=\left\{x \in \mathbb{R}^{n}: \sum_{i}\left(x_{i} / a_{i}\right)^{2}=1\right\}
$$

be the minimum ellipsoid of $\Omega$ with $0<a_{1} \leq \cdots \leq a_{n}$. Then we have, when choosing the origin properly, $\frac{1}{n} E \subset \Omega \subset E$. Let $\mathcal{M}^{\prime}$ be the projection of $\mathcal{M}^{+}$on the subspace $\left\{x_{k+1}=\cdots=x_{n}=0\right\}$. Then we have

$$
f\left(\kappa^{\prime}\left(\mathcal{M}^{\prime}\right)\right) \leq f\left(\kappa\left(\mathcal{M}^{+}\right)\right)
$$

where $\kappa^{\prime}=\left(\kappa_{1}, \cdots, \kappa_{k}\right)$ are the principal curvatures of $\mathcal{M}^{\prime}$. Since $f\left(\kappa^{\prime}\right)=$ $\sigma_{k}\left(\kappa^{\prime}\right)$ is the Gauss curvature of $\mathcal{M}^{\prime}$, if the linear function $a$ is given by (3.3) for some sufficiently large $\alpha>0$, we have

$$
a_{1} \cdots a_{k}>>1
$$

Namely $a_{i_{1}} \cdots a_{i_{k}}>>1$ for any $1 \leq i_{1}<\cdots<i_{k} \leq n$.

Next we consider the piece $\mathcal{M}^{-}$, which satisfies equation (3.5) with $\alpha \geq 0$. Let $\mathcal{M}^{-}$be the graph of a convex function $u_{0}$. For any $1 \leq i_{1}<\cdots<i_{k} \leq$ $n$, let $\kappa_{i_{1} \cdots i_{k}}$ denote the Gauss curvature of the restriction of $\mathcal{M}^{-}$on the subspace determined by the $\left\{x_{i_{1}}, \cdots, x_{i_{k}}\right\}$-axes. Then

$$
\sum_{i_{1}<\cdots<i_{k}} \kappa_{i_{1} \cdots i_{k}} \geq \sigma_{k}(\kappa) \geq \varphi
$$

Hence there exists an index set $\left\{1 \leq i_{1}<\cdots<i_{k} \leq n\right\}$ such that $\kappa_{i_{1} \cdots i_{k}} \geq \inf \varphi / C_{n, k}$ on a set $G \subset \frac{1}{n} E$ with Lebesgue measure $|G| \geq|E| /\left(n^{n} C_{n, k}\right)$, where $C_{n, k}=\frac{n !}{k !(n-k) !}$. There is no loss of generality in assuming that $\left\{i_{1}, \cdots, i_{k}\right\}=\{1, \cdots, k\}$. Then there exists a point $y=\left(0, \cdots, 0, y_{k+1}, \cdots, y_{n}\right) \in \frac{1}{n} E$ such that the Gauss curvature of $\mathcal{M}^{\prime}=\mathcal{M}^{-} \cap P$ is larger than $\inf \varphi / C_{n, k}$ on a set $G^{\prime} \subset \Omega \cap P$ with Lebesgue measure $|G| \geq \delta_{0} a_{1} \cdots a_{k}$ for some $\delta_{0}>0$, where $P$ is the hyperplane $\left\{x_{k+1}=y_{k+1}, \cdots, x_{n}=y_{n}\right\}$. Let $\mathcal{M}^{\prime}$ be the graph of a convex 
function $u^{\prime}$. Then

$$
\frac{\operatorname{det} D_{x^{\prime}}^{2} u^{\prime}}{\left(1+\left|D u^{\prime}\right|^{2}\right)^{(k+2) / 2}} \geq \inf \varphi / C_{n, k} \quad \text { in } \quad G^{\prime}
$$

where $x^{\prime}=\left(x_{1}, \cdots, x_{k}\right)$. Taking integration we find that $\left|G^{\prime}\right| \leq C$, which is in contradiction with (3.19).

For the curvature (1.2), we have

$$
\frac{1}{n}\left[\sigma_{1}(\kappa)\right]^{2} \leq \kappa_{1}^{2}+\cdots+\kappa_{n}^{2} \leq\left[\sigma_{1}(\kappa)\right]^{2} .
$$

As we need to prove (3.2) uniformly for any $t \in[0,1]$, we need to replace the operator in (2.4) by

$$
F_{t}\left[\nabla^{2} h+h I\right]=(1-t) \hat{h}(\lambda)+t \hat{f}(\lambda),
$$

where $\hat{h}(\lambda)=1 / \sigma_{1}(\kappa)$. For the operator in (3.21), the argument in $\S 2$ is still valid.

We remark that for the estimate (3.2), both proofs above may be extended to other Weingarten curvatures. But whether (3.2) holds for any curvature satisfying $[\mathrm{F} 1]-[\mathrm{F} 4]$ is still unknown.

\section{Verification of condition (A).}

Lemma 4.1. For any smooth, positive function $\varphi$ on $S^{n}$, there exists a unique vector $y \in \mathbb{R}^{n+1}$ such that

$$
\int_{S^{n}} x_{i} \varphi(x) e^{y \cdot x} d x=0 \quad \forall i=1, \cdots, n+1 .
$$

Lemma 4.1 follows from Theorem A in [5]. It can also be proved directly as follows.

Proof. Let $T_{0}: \mathbb{R}^{n+1} \rightarrow B_{1}(0)$ be a mapping such that $T_{0}(0)=0$ and $\forall x \neq 0$,

$$
T_{0}(x)=\frac{2}{\pi} \frac{x}{|x|} \operatorname{arctg}|x|
$$

Next we define a mapping $T_{1}: \mathbb{R}^{n+1} \rightarrow \mathbb{R}^{n+1}$ such that $\forall y \in \mathbb{R}^{n+1}$,

$$
T_{1}(y)=\left(\xi_{1}(y), \cdots, \xi_{n+1}(y)\right), \quad \xi_{i}(y)=\int_{S^{n}} x_{i} \varphi(x) e^{y \cdot x} .
$$


Then there exists a constant $R>0$ such that

$$
\left\langle y, T_{1}(y)\right\rangle>0 \quad \text { if }|y| \geq R
$$

where $\langle x, y\rangle=x \cdot y$ is the inner product. We modify $T_{1}$ by letting $\hat{T}_{1}(y)=$ $T_{1}(y)$ if $|y| \leq R$ and

$$
\hat{T}_{1}(y)=t \frac{y}{|y|}\left|T_{1}(y)\right|+(1-t) T_{1}(y)
$$

if $|y| \geq R$, where $t=\frac{2}{\pi} \operatorname{arctg}(|y|-R)$. Then we have

$$
\frac{\hat{T}_{1}(y)}{\left|\hat{T}_{1}(y)\right|} \rightarrow e \quad \text { if }|y| \rightarrow \infty \text { and } \frac{y}{|y|} \rightarrow e
$$

for any unit vector $e \in S^{n}$.

Denote $T=T_{0} \cdot \hat{T}_{1} \cdot T_{0}^{-1}$. Then $T$ is a mapping from $B_{1}$ to $B_{1}$. By (4.3) we can extend $T$ to $S^{n}=\partial B_{1}$ continuously such that $T$ is the identity mapping on $S^{n}$. Hence we must have $T\left(B_{1}\right)=B_{1}$ and so there is a point $y$ such that $T(y)=0$, namely $\hat{T}_{1}(y)=0$. By $(4.2), \hat{T}_{1}(z) \neq 0$ if $|z| \geq R$. Hence $|y|<R$ and so (4.1) holds at $y$.

For the uniqueness, we compute the Jacobi matrix

$$
\partial_{y_{i}} \xi_{j}(y)=\int_{S^{n}} x_{i} x_{j} \varphi(x) e^{y \cdot x}
$$

The Jacobi matrix is symmetric. We claim it is positive definite. Indeed, let $A=\left\{a_{i j}\right\}$ be an orthogonal matrix such that $A\left\{\partial_{y_{i}} \xi_{j}(y)\right\} A^{\prime}$ is diagonal at a given point $y$. Denote $\widetilde{y}=A y$ and

$$
\widetilde{\xi}_{i}(\widetilde{y})=\int_{S^{n}} \widetilde{x}_{i} \varphi\left(A^{\prime} \widetilde{x}\right) e^{\widetilde{y} \cdot \widetilde{x}}
$$

Then

$$
\begin{aligned}
\left\{\partial_{\widetilde{y}_{i}} \widetilde{\xi}_{j}(\widetilde{y})\right\} & =\left\{\int_{S^{n}} \widetilde{x}_{i} \widetilde{x}_{j} \varphi\left(A^{\prime} \widetilde{x}\right) e^{\widetilde{y} \cdot \widetilde{x}}\right\} \\
& =\left\{\int_{S^{n}} \widetilde{x}_{i} \widetilde{x}_{j} \varphi(x) e^{y \cdot x}\right\}=A\left\{\partial_{y_{i}} \xi_{j}(y)\right\} A^{\prime}
\end{aligned}
$$

Hence it is positively definite. If $y_{0}$ and $y_{1}$ are two points such that $\xi_{i}\left(y_{0}\right)=$ $\xi_{i}\left(y_{1}\right)=0$ for $i=1, \cdots, n+1$, let $y_{t}=(1-t) y_{0}+t y_{1}$. Then

$$
\xi\left(y_{1}\right)-\xi\left(y_{0}\right)=\int_{0}^{1}\left\{\partial_{y_{i}} \xi_{j}\left(y_{t}\right)\right\} d t\left(y_{1}-y_{0}\right) \neq 0 .
$$


We reach a contradiction.

We will first show that there exists a family of continuous, positive functions $\left\{\varphi_{t}: t \in[0,1]\right\}$ verifying Condition $(\mathrm{A})$.

Let $g$ be a function on $\mathbb{R}^{1}$, given by

$$
g(t)= \begin{cases}t & \text { if } t>0 \\ 0 & \text { if } t \leq 0\end{cases}
$$

For any $z \in \mathbb{R}^{n+1}$, denote

$$
h_{z}(x)=g(z \cdot x)
$$

Let $\varphi$ be a smooth positive function on the unit sphere $S^{n}$ such that $\varphi^{-1 / k}$ is convex. Denote $\psi=\varphi^{-1 / k}$ and $\psi_{z}=\psi+h_{z}$. Then $\psi_{z}$ is convex. Denote $\varphi_{z}=\psi_{z}^{-k}$ and

$$
\xi_{i}(z)=\int_{S^{n}} x_{i} \varphi_{z}(x) d x \quad i=1, \cdots, n+1 .
$$

Lemma 4.2. There exists a unique $z \in B_{1}(0)$ such that $\xi_{i}(z)=0 \forall i=$ $1, \cdots, n+1$.

Proof. First we prove the existence. Let $T_{0}$ be the mapping given in the proof of Lemma 4.1. We define a mapping $T_{1}: \mathbb{R}^{n+1} \rightarrow \mathbb{R}^{n+1}$ such that for any $z \in \mathbb{R}^{n+1}, y=T_{1}(z)$ is the unique point such that $\int_{S^{n}} x_{i} \varphi_{z}(x) e^{y \cdot x}=0$ for all $i=1, \cdots, n+1$. Denote $T=T_{0} \cdot T_{1} \cdot T_{0}^{-1}$. Then $T$ is a mapping from $B_{1}$ to $B_{1}$.

We claim that $T$ can be extended continuously to $\partial B_{1}$ such that it is the identity mapping on $\partial B_{1}$, from which the existence part of Lemma 4.2 follows. Indeed, let $z_{0} \in \partial B_{1}$ be a boundary point. Choosing a proper coordinate system we may suppose that $z_{0}=(1,0, \cdots, 0)$. Let $\left\{z_{k}\right\}$ be a sequence in $B_{1}$ converging to $z_{0}$, and denote $\hat{z}_{k}=T_{0}^{-1}\left(z_{k}\right)$ and $y^{k}=$ $\left(y_{1}^{k}, \cdots, y_{n+1}^{k}\right)=T_{1}\left(\hat{z}_{k}\right)$. Then at any point $x$ in the hemisphere $S^{n} \cap\left\{x_{1}>\right.$ $0\}, \psi_{\hat{z}_{k}}(x) \rightarrow \infty$ and $\varphi_{\hat{z}_{k}}(x) \rightarrow 0$ as $k \rightarrow \infty$. On the other hand, for any $\delta>0$, there is a $k_{0}>1$ such that $\varphi_{\hat{z}_{k}}(x) \geq \inf _{S^{n}} \varphi$ on $S^{n} \cap\left\{x_{1}<-\delta\right\}$ for all $k \geq k_{0}$. It follows that $\left|y_{i}^{k}\right| \leq C$ for any $i=2, \cdots, n+1$ and $k \geq k_{0}$, and $y_{1}^{k} \rightarrow \infty$ as $k \rightarrow \infty$. Hence $T\left(z_{k}\right) \rightarrow(1,0, \cdots, 0)$.

Next we prove the uniqueness. Suppose to the contrary that there exist $z_{0} \neq z_{1}$ such that $\xi_{i}\left(z_{0}\right)=\xi_{i}\left(z_{1}\right)=0$ for all $i=1, \cdots, n+1$. If the origin lies on the line segment $z_{0} z_{1}$, namely if $z_{1}=\alpha z_{0}$ for some constant $\alpha$, we 
may suppose by choosing a proper coordinate system that $z_{i}=\left(a_{i}, 0, \cdots, 0\right)$ with $a_{0}<a_{1}$. Denote $z_{t}=t z_{1}+(1-t) z_{0}$. Then

$$
\frac{d}{d t} \xi_{1}\left(z_{t}\right)=-k \int_{S^{n}} x_{1} \psi_{z_{t}}^{-k-1}\left(\frac{d}{d t} h_{z_{t}}\right)=-k \int_{S_{z_{t}}^{n}} x_{1}^{2} \psi_{z_{t}}^{-k-1},
$$

where $S_{z}^{n}=\left\{x \in S^{n}: x \cdot z>0\right\}$. Hence $\xi_{1}\left(z_{t}\right)$ is strictly decreasing in $t$, which is in contradiction with $\xi_{1}\left(z_{0}\right)=\xi_{1}\left(z_{1}\right)=0$.

If the origin is not on the line segment $z_{0} z_{1}$, then as in the proof of Lemma 1, the Jacobi matrix

$$
\partial_{z_{i}} \xi_{j}(z)=-k \int_{S_{z}^{n}} x_{i} x_{j} \psi_{z}^{-k-1}, \quad z \neq 0,
$$

is symmetric and negative definitive, and

$$
\xi\left(z_{1}\right)-\xi\left(z_{0}\right)=\int_{0}^{1}\left\{\partial_{z_{i}} \xi_{j}\left(z_{t}\right)\right\} d t\left(z_{1}-z_{0}\right) \neq 0 .
$$

We also reach a contradiction.

Lemma 4.3. Let $\varphi$ be a function as in Theorem 1.2. Then there exists a family of continuous positive functions verifying condition (A).

Proof. Denote $\psi^{0}(x)=|x|$ and $\psi^{t}(x)=(1-t) \psi^{0}(x)+t \psi(x)$. Denote $\psi_{z}^{t}=$ $\psi^{t}+h_{z}$ and $\varphi_{z}^{t}=\left(\psi_{z}^{t}\right)^{-k}$. Let

$$
\xi_{i}^{t}(z)=\int_{S^{n}} x_{i} \varphi_{z}^{t}(x) d x \quad i=1, \cdots, n+1
$$

By Lemma 4.2 , for any $t \in(0,1)$, there is a unique $z_{t}$ such that $\xi_{i}^{t}\left(z_{t}\right)=0$ for all $i=1, \cdots, n+1$. The uniqueness in Lemma 4.2 also implies that $z_{t}$ is continuous in $t$. This is because if there are two sequences $t_{k} \rightarrow t_{0} \in[0,1]$ and $\hat{t}_{k} \rightarrow t_{0}$, then by the uniqueness, both $z_{t_{k}}$ and $\hat{z}_{t_{k}}$ converge to the same limit. Denote $\varphi_{t}=\varphi_{z_{t}}^{t}$. Then $\left\{\varphi_{t}\right\}$ is a family of continuous positive functions verifying condition (A).

Verification of Condition ( $A$ ). In Lemma 4.3 we have proved the existence of continuous $\varphi_{t}$ such that $\varphi_{t}^{-1 / k}$ is convex and (1.10) holds. To prove the existence of smooth $\varphi_{t}$ satisfying these conditions, we need some modifications of the definition of the function $h_{z}$ in (4.5). 
Let $\left\{g_{t}\left(x_{1}\right): x_{1} \in[-1,1], t \geq 0\right\}$ be a family of smooth, nonnegative functions satisfying the following conditions:

(i) $g_{0} \equiv 0, g_{t}$ is increasing in $t$, and $g_{t}=\varepsilon_{t}+t x_{1}$ when $x_{1}>0$, where $\varepsilon_{t} \leq \min \left(t^{2}, \varepsilon^{*}\right)$ for some sufficiently small $\varepsilon^{*}>0$.

(ii) $g_{t}$ is increasing in $x_{1}$, and is convex in the sense that $\hat{g}_{t}(x)=g_{t}\left(x_{1}\right)$ is convex as a function on $S^{n}$. Note that the convexity of $g_{t}$ is equivalent to that $\frac{d^{2}}{d \theta^{2}} g_{t}(\cos \theta)+g_{t} \geq 0$.

(iii) $\frac{\partial}{\partial t} g_{t}\left(x_{1}\right)<\varepsilon^{*}$ when $x_{1} \leq 0$.

Denote

$$
h_{z}(x)=g_{|z|}\left(\frac{z}{|z|} \cdot x\right)
$$

The function $h_{z}$ in (4.8) is a small perturbation of that in (4.5). Indeed, if $\varepsilon_{t} \equiv 0$, then (4.5) and (4.8) are the same. Note that the smoothness of $g_{t}$ implies that $\varepsilon_{t}>0$ if $t>0$.

Define $\psi_{z}$ and $\varphi_{z}$ as in Lemma 4.2. With $h_{z}$ given in (4.8), the existence part in Lemma 4.2 can be proved in the same way. By assumptions (i)-(iii), the uniqueness part in the proof of Lemma 4.2 is also valid. Hence from the proof of Lemma 4.3, we obtain a family of smooth $\varphi_{t}$ which verifies Condition (A).

\section{References.}

[1] A.D. Aleksandrov, On the curvature of surfaces (Russian) Vestnik Leningrad. Univ., 21(1966), 5-11.

[2] B. Andrews and X.N. Ma, The Christoffel-Minkowski problem II: A parabolic approach, Preprint.

[3] H. Busemann, Convex surfaces, Interscience Publishers, New York, 1958.

[4] L. Caffarelli, L. Nirenberg, J. Spruck, Nonlinear second order elliptic equations IV, star-shaped compact Weingarten hypersurfaces, Current Topics in Partial Differential Equations, Ohya, Kasahara and Shimakura (eds), Tokyo, 1985, 1-26.

[5] K.-S. Chou and X.-J. Wang, The logarithmic Gauss curvature flow, Ann. Inst. H. Poincare, Anal. Non Lineaire, 17(2000), 733-751.

[6] E.B. Christoffel, Uber die Bestimmung der Gestalt einer krummen Oberfläche durch lokale messungen auf derselben, J. Reine Angew. Math., 64(1865), 193-209. 
[7] S.-Y. Cheng and S.-Y. Yau, On the regularity of the solution of the $n$ dimensional Minkowski problem, Comm. Pure Applied Math., 29(1976), 495-516.

[8] K. Ecker and G. Huisken, Immersed hypersurfaces with constant Weingarten curvature, Math. Ann., 283(1989), 329-332.

[9] W.J. Firey, Christoffel problem for general convex bodies, Mathematik, 15(1968), 7-21.

[10] C. Gerhardt, Closed Weingarten hypersurfaces in Riemannian manifolds. J. Differ. Geom. 43 (1996), 612-641.

[11] C. Gerhardt, Hypersurfaces of prescribed Weingarten curvature, Math Z., 224(1997), 167-194.

[12] D. Gilbarg and N.S. Trudinger, Elliptic partial differential equations of second order, Springer, Berlin, Heidelberg, Second Edition, 1983.

[13] B. Guan and P. Guan, Convex hypersurfaces of prescribed curvature, Ann Math., 156(2002), 655-673.

[14] P. Guan and X.N. Ma, The Christoffel-Minkowski problem I: Convexity of solutions of a Hessian equation, Invent. Math., 151(2003), 553-577.

[15] Y.Y. Li, Group invariant convex hypersurfaces with prescribed GaussKronecker curvature. Multidimensional complex analysis and partial differential equations (Sao Carlos, 1995), 203-218, Contemp. Math. 205.

[16] H. Minkowski, Volumen und oderfläche, Math. Ann., 57(1903), 447-495.

[17] A.V. Pogorelov, The Minkowski multidimensional problem, J. Wiley, New York, 1978.

[18] R. Schneider, Convex bodies, the Bruun-Minkowski theory, Cambridge Univ. Press, 1993.

[19] W.M. Sheng, J. Urbas, and X.-J. Wang, Interior curvature bounds for a class of curvature equations, Duke Math. J., to appear.

[20] N.S. Trudinger, The Dirichlet problem for the prescribed curvature equations, Arch. Rational Mech. Anal., 111 (1990), 153-179.

[21] J. Urbas, An interior curvature bound for hypersurfaces of prescribed $k$-th mean curvature, J. Reine Angew. Math., 519(2000), 41-57. 


\author{
Weimin Sheng \\ Department of Mathematics \\ ZHEJIANG UNIVERSITY \\ Hangzhou 310028, China \\ weimins@zju.edu.cn \\ Neil S. Trudinger \\ Centre for Mathematics and Its Applications \\ Australian National University \\ Canberra, ACT 0200, Australia \\ neil .trudinger@maths . anu . edu . au \\ XU-Jia Wang \\ Centre for Mathematics and its Applications \\ Australian National University \\ Canberra ACT 0200, Australia \\ wang@maths . anu . edu . au
}

